\title{
Probing the Local Chemical and Structural Ordering of Iron Oxyfluoride
}

\author{
Dong $\mathrm{Su}^{1}$, Sung-Wook Kim ${ }^{2}$, Kai $\mathrm{He}^{1}$, Nathalie Pereira ${ }^{3}$, Glenn G. Amatucci ${ }^{3}$, Jason Graetz ${ }^{2}$, and Feng \\ Wang $^{2}$
}

1. Center for Functional Nanomaterials, Brookhaven National Laboratory, Upton, New York, 11973,USA

2. Sustainable Energy Technologies Department, Brookhaven National Laboratory, Upton, New York, 11973, USA

3. Dept of Materials Science and Engineering, Rutgers University, North Brunswick, NJ 08902

Scanning transmission electron microscopy (STEM)-electron energy loss spectroscopy (EELS) has been used for studying local structure of the energy related materials. High resolution (Cs-corrected) STEM is regarded as a promising technique to observe morphological evolution and atomic arrangement inside single particles. Combining with EELS technique, one could probe the local chemical structure therefore to give the high spatial resolution information on the chemical evolution, which information could not be obtained by other characterization techniques, such as X-ray diffraction (XRD) and X-ray absorption spectroscopy (XAS).

In the iron fluorite system used for the cathode for Li-ion battery, conversion reaction behavior of $\mathrm{FeF}_{2}$ was investigated using STEM-EELS technique.[1-2] The importance of the local morphological evolution on the reversibility was identified by comparing highly reversible $\mathrm{FeF}_{2}$ and irreversible $\mathrm{CuF}_{2}$. $\mathrm{Fe} 0$ nanoparticles $(<5 \mathrm{~nm})$ interconnected each other to form bicontinuous network inside LiF matrix while larger $\mathrm{Cu} 0$ particles $(5-12 \mathrm{~nm})$ were isolated by surrounding $\mathrm{LiF}$ after lithiation. In contrast to simple phase behavior of FeF2 $\left(\mathrm{FeF}_{2}+2 \mathrm{Li}_{+}+\mathrm{e}_{-}=2 \mathrm{LiF}+\mathrm{FeO}\right)$, multiple phase coexist together in $\mathrm{FeO}_{1-\delta} \mathrm{F}_{1+\delta}$ cathode during cycling, implying the complexity in the morphological and chemical evolution during the reaction.[3] The separation of O-based phase (rocksalt Li-Fe-O(-F)) and F-based phase ( $\mathrm{LiF}$, amorphous $\mathrm{FeF}_{2}$ ) was identified from the literature, but how the $\mathrm{O} / \mathrm{F}$-based phases are distributed inside the single particle is not clear yet. In addition, valence state of $\mathrm{Fe}$ and its distribution in $\mathrm{FeO}_{1-\delta} \mathrm{F}_{1+\delta}$ during cycling is a critical issue to understand the reaction. Since Fe atom has variety of valence states (e.g., $\mathrm{Fe}_{0}$, $\mathrm{Li}-\mathrm{Fe}-\mathrm{O}$, amorphous $\mathrm{FeF}_{2}$ ), the Fe valence distribution could inform the phase distribution inside the single particle as a function of $\mathrm{Li}$ content. In this respect, high resolution characterization is expected to be the most suitable analytical tool to study the single particle behavior of $\mathrm{FeO}_{1-\delta} \mathrm{F}_{1+\delta}$ cathode to complement the bulk measurement techniques in the literatures.

Here, we studied morphological and chemical evolution of well-ordered ellipsoidal $\mathrm{FeO}_{0.7} \mathrm{~F}_{1.3}$ nanoparticle during cycling to understand the impact of $\mathrm{O}$-substitution to $\mathrm{FeF}_{2}[4]$. Morphological evolution depending on Li content during cycling was traced by HAADF imaging. Spatial distribution of constituent elements (i.e., Li, Fe, O, F) and valence state of Fe at pristine, discharged (1.5 V) and recharged $(4.5 \mathrm{~V}) \mathrm{FeO}_{0.7} \mathrm{~F}_{1.3}$ single particle was identified via high resolution EELS $(<1 \mathrm{~nm})$ to track the $\mathrm{O} / \mathrm{F}$ distribution, redox reaction of $\mathrm{Fe}$, and corresponding atomic rearrangement during the $\mathrm{Li}$ storage/release reaction. 


\section{References}

[1] F. Wang, et al., J. Am. Chem. Soc. 133, 18828(2011)

[2] F. Wang, et al., Nature Commun., 3. 1201(2012)

[3] K. M. Wiaderek, et al., J. Am. Chem. Soc. 135, 4070(2013)

[4] Sung-Wook Kim, Natasha Chernova, Stan M. Whittingham, Jason Graetz, Dong Su and Feng Wang, Unpublished

[5] Research carried out at the Center for Functional Nanomaterials, BNL, which is supported by the DOE, Office of Basic Energy Sciences, under Contract No. DE-AC02-98CH10886. This work was supported by the Northeastern Center for Chemical Energy Storage, an Energy Frontier Research Centre funded by the U.S. DOE, BES under award number DE-SC0001294.
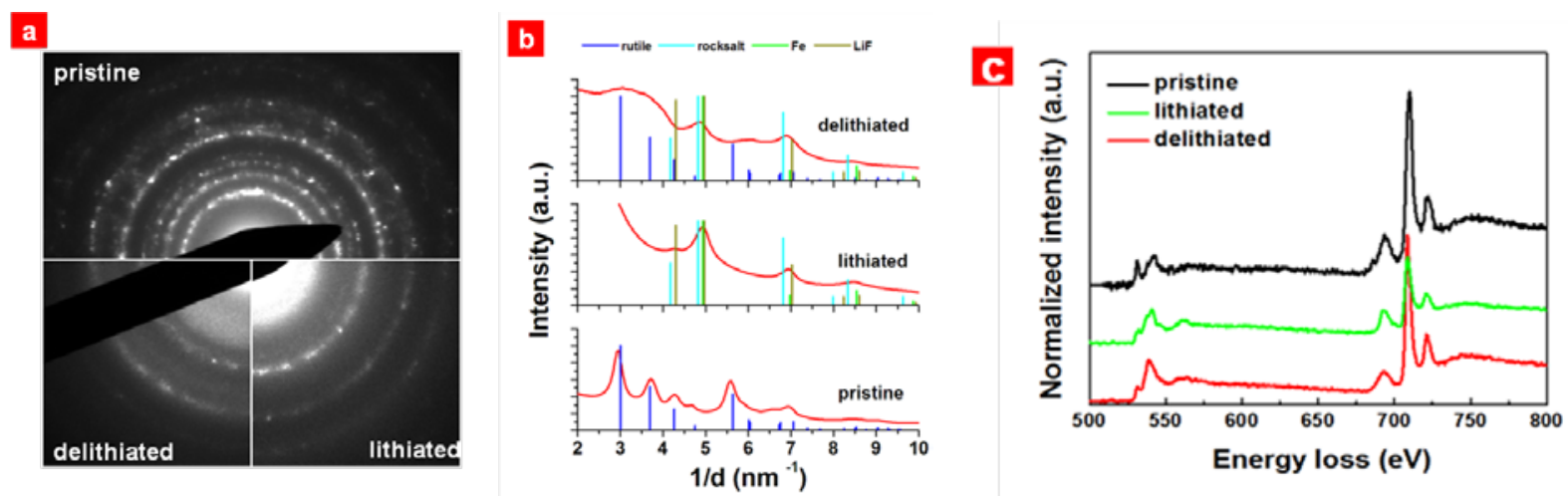

Figure1 Diffraction pattern (a,b) and EELS spectra(c) of pristine, delithiated and lithiated samples.


Figure 2 (a)ADF image of a $\mathrm{FeO}_{0.7} \mathrm{~F}_{1.3}$ nanoparticle at the lithiated state. (b) Selected area EELS spectra from the core and shell region. (c) Elemental distribution of Fe (green) and $\mathrm{Li}$ (red) (scale bar: $5 \mathrm{~nm}$ ) of FeO0.7 $\mathrm{F}_{1.3}$ at lithiated state. 